\title{
Gestión óptima de la potencia eléctrica en una microrred conectada, basada en el algoritmo genético para optimización multiobjetivo MOGA
}

\section{Optimal power management in a grid-connected microgrid, based on Multi- Objective Genetic Algorithm MOG}

\author{
F.A. Zúñiga-Cortés ${ }^{1}$, E.F Caicedo-Bravo ${ }^{2}$, D.M López-Santiago ${ }^{3}$ \\ ${ }^{1}$ Ing. electrónico Univalle, Cali, Colombia, fabian.zuniga@ correounivalle.edu.co; ${ }^{2} \mathrm{PhD}$ en Informática Industrial, Univalle, Cali, \\ Colombia, eduardo.caicedo@ correounivalle.edu.co; ${ }^{3}$ MSc en Ciencias de la Computación, Univalle, Cali, Colombia, \\ danny.lopez@correounivalle.edu.co
}

Recibido: dic 12, 2014. Aceptado: may 25, 2016. Versión final: may 25, 2016

\begin{abstract}
RESUMEN
Las microrredes son un concepto revolucionario que enfrenta algunos problemas de los grandes sistemas eléctricos centralizados. Aunque este concepto promete numerosos beneficios, también presenta nuevos desafíos en materia de control y operación. Uno de los desafíos más importantes es gestionar, de manera óptima, la energía producida por las microrredes. Este artículo presenta una estrategia de gestión de potencia en una microrred conectada, basada en el algoritmo MOGA. La estrategia se formula con base en dos objetivos conflictivos entre sí: la reducción de los costos operacionales de la microrred y la reducción de la cantidad de emisiones de gases contaminantes. Los resultados de simulación muestran los beneficios de usar la estrategia de gestión basada en MOGA, en comparación con una estrategia de importación total de la red principal, lo cual hace a MOGA una técnica competitiva para integrar el núcleo optimizador de un sistema de gestión de energía en microrredes.
\end{abstract}

Palabras clave: algoritmos evolutivos de optimización multiobjetivo (MOEA), algoritmo genético multiobjetivo (MOGA), gestión óptima de potencia, microrred, optimización multiobjetivo, sistema de gestión de energía (EMS).

\begin{abstract}
Microgrids are a revolutionary concept to face some problems of the big and centralized power systems. Although emerging concept promises to offer numerous benefits, also presents new challenges in control and operation. One of the most important challenges is to make optimal power management into the microgrid. This power management must ensure maximum use of available resources; at same time it must reduce environmental contamination. Based on Multi-Objective Genetic Algorithm MOGA, this paper presents one strategy to make optimal power management in a grid-connected microgrid. The strategy runs over two conflictive objectives: to reduce microgrid operational cost and greenhouse emissions. Simulation results show benefits using the MOGA strategy, in comparison with a strategy of full importation from main grid. In this manner, the proposed strategy is a good option to build the optimizer kernel of energy management system EMS in microgrids.
\end{abstract}

Keywords: energy management system (EMS), microgrid, multi-objective optimization, multi-objective genetic algorithm (MOGA), multi-objective optimization evolutionary algorithms (MOEA), optimal power management.

Este artículo puede compartirse bajo la licencia CC BY-ND 4.0 (https://creativecommons.org/licenses/by-nd/4.0/) F.A. Zúñiga-Cortés, E.F Caicedo-Bravo y D.M López-Santiago. "Gestión óptima de la potencia eléctrica en una microrred conectada, basada en el algoritmo genético para optimización multiobjetivo MOGA”, UIS Ingenierias, vol. 15, no. 2, pp. 17-33, jul-dic 2016, doi: 10.18273/revuin.v15n2-2016002. 


\section{INTRODUCCIÓN}

El actual sistema de energía eléctrica, de acuerdo con [1] presenta una serie de problemas entre los que cabe mencionar que: su arquitectura centralizada relega conceptos de eficiencia, sostenibilidad y flexibilidad; este hace uso de tecnologías que fueron concebidas antes de la mitad del siglo pasado; presenta altas emisiones de gases contaminantes; los actuales marcos comerciales y de regulación heterogéneos no se ajustan a este modelo convencional. Estos, entre otros problemas, implican algunos cambios en los procesos de generación, transmisión y distribución de la energía eléctrica (GTD). En este sentido, la generación a pequeña escala, en particular el concepto de generación distribuida (GD) a través de recursos energéticos distribuidos (DER) y especialmente fuentes distribuidas de energías alternativas (AEDG), ha tenido una amplia acogida dentro de las soluciones que se plantean, entre otros, [2].

Para capitalizar las ventajas de la GD de una manera eficiente, y teniendo en cuenta la necesidad de sistemas eléctricos más flexibles, se ha evolucionado al concepto de microrredes, las cuales de una manera descentralizada buscan la penetración y organización de pequeñas y diversas unidades de generación en media y baja tensión. En un máximo aprovechamiento de los recursos energéticos de la microrred, se debe realizar una correcta planificación de la solución energética, haciendo énfasis en la importancia de que esta vaya acompañada de una adecuada optimización de los recursos. Al complementarse estos dos aspectos (gestión y optimización) será posible obtener una solución energética integral.

En el futuro contexto de la explotación de microrredes, las decisiones importantes deberán ser tomadas de la manera más eficientemente posible y en horizontes de corto plazo, por lo que los métodos de Inteligencia Computacional (IC) serán de gran importancia en este campo. Aunque el papel que juega la IC en la gestión de potencia eléctrica de microrredes es relativamente innovador, existe hasta la fecha algunos trabajos de investigación que buscan dar respuesta a este problema. Estos trabajos han demostrado la eficacia de los métodos de IC para ser aplicados en sistemas de potencia como las microrredes.

A través del algoritmo genético de ordenación no dominada II NSGAII, [3] hace frente al problema del despacho óptimo de DER, al buscar la minimización de los costos de generación, las emisiones de carbono y las pérdidas en las líneas. Para programar la operación entre microrredes vecinas interconectadas, es presentado un EMS, en [4], cuya arquitectura hace uso de la función de una red neuronal artificial ANN para predecir tanto la carga como la disponibilidad de los DER en las microrredes.
Un EMS basado en optimización por enjambre de partículas PSO que busca minimizar los costos de operación de una red de distribución y de una microrred conectada a esta red, es presentado en [5]. El estudio considera una auténtica red de distribución con 20310 consumidores y 548 DER. La Universidad del Valle en Colombia, con sus departamentos de ingeniería eléctrica, y de sistemas y computación, como se muestra en [6], se propone una estrategia basada en los algoritmos NSGA II y MOPSO para la gestión óptima de potencia eléctrica en un modelo de microrred, sobre la cual se realiza la gestión de DER teniendo en cuenta los costos de operación y las emisiones de los mismos. Por otro lado, el trabajo también ofrece un análisis comparativo del desempeño de ambos algoritmos, con el fin de verificar la prevalencia de una técnica sobre la otra.

En [7] se expone una metodología para determinar la disponibilidad y el costo del recurso energético entregado por una microrred con generación eólica y solar, y conexión a la red eléctrica principal. La metodología propuesta pretende un suministro fiable de energía con la menor inversión económica, para lo cual hace uso del Algoritmo MOGA como método de optimización. En [8] se presenta un algoritmo híbrido basado en PSO y el algoritmo de búsqueda de patrón, para evaluar y optimizar la operación de una microrred conectada a las redes de gas y electricidad. Los autores proponen cuatro indicadores claves de desempeño KPIs: de costos económicos, fiabilidad, emisiones y calidad de la energía; y a partir de ellos buscan resultados óptimos en la generación de energía.

Este documento está organizado en las siguientes secciones: la sección 2 expone el problema de la gestión de potencia eléctrica en un ambiente de microrredes; la sección 3 presenta una abstracción de una microrred, la cual está conformada por DER, AEDG, un sistema DS, y un sistema de gestión de energía EMS; la sección 4 presenta el modelo de costos y emisiones ambientales involucrado en la operación de la microrred, y además tiene en cuenta algunas restricciones relacionadas con la demanda de potencia y el rango de trabajo de los DER. La sección 5 exhibe el esquema de la estrategia de gestión, basada en MOGA. La sección 6 presenta un análisis de los resultados obtenidos. Finalmente, se presentan las conclusiones de los resultados obtenidos a lo largo del desarrollo del trabajo.

\section{GESTIÓN DE POTENCIA ELÉCTRICA EN MICRORREDES}

El concepto de microrredes trae consigo un gran número de retos económicos, comerciales y técnicos, que deben ser afrontados de cara a este nuevo modelo. En particular, uno de estos retos es la gestión de potencia eléctrica [9] y la complejidad que implica es mucho mayor si se involucran diferentes fuentes de energía, dispositivos de almacenamiento y cargas controlables. 
Esto se puede soslayar solo si las diferentes alternativas de generación se integran adecuadamente, y se realiza una planificación de los tiempos de generación y consumo, a través de un EMS que logre un máximo aprovechamiento de los recursos existentes [10], respetando al mismo tiempo las diversas restricciones de las fuentes de generación y las restricciones del sistema, por ejemplo: la disponibilidad de los recursos, el balance y los límites de potencia, los factores de estabilidad, los niveles de frecuencia y tensión, la demanda de energía de las cargas, el modo de operación de la microrred (conectado o aislado), entre otros.

El interés de producir energía de una manera distribuida en un entorno de microrredes está motivado por la posibilidad de satisfacer diferentes objetivos estratégicos de importancia en la actualidad para el sistema de energía, [11], tales como minimizar el impacto ambiental en la explotación de los generadores, incrementar la flexibilidad de los actores energéticos, mejorar la competitividad del mercado de la electricidad, reducir las pérdidas técnicas y comerciales, mejorar la eficiencia energética, etc. El EMS de una microrred es un ejemplo de un marco de toma de decisiones automatizado; cuando estas decisiones se toman hacia un conjunto de objetivos, como los mencionados anteriormente se debe encontrar una solución de lo que en [3] se conoce como un problema combinatorio multiobjetivo y multirestricción. En el proceso de búsqueda de la mejor solución disponible, este tipo de problemas se incorpora dentro un campo de análisis de optimización. Uno de los conceptos ligados a este campo es el concepto de óptimo de Pareto, el cual representa una solución que considera un estado de satisfacción para un objetivo que no se puede mejorar más sin bajar la satisfacción de otro de los objetivos.

Para abordar un problema tan complejo como es la gestión de potencia eléctrica en una microrred se requieren técnicas nuevas e innovadoras, dado que las técnicas determinísticas pueden ser costosas computacionalmente y aun así no derivar soluciones de Pareto. Bajo esta premisa, se puede dar uso al potencial de algunas de las técnicas de IC que tengan la capacidad de hacerle frente a problemas no lineales y de gran dimensión; que operen sobre sobre un conjunto de posibles soluciones; que garanticen que las restricciones no sean violadas; y que puedan abordar varios objetivos simultáneamente, al tiempo que requieran menos recursos computacionales para derivar soluciones en menos tiempo, en comparación con los métodos determinísticos clásicos [5]. Dentro de las técnicas de IC se debe prestar especial atención a los Algoritmos Evolutivos de Optimización Multiobjetivo o MOEA, cuyas características los hacen atractivos para la elaboración de soluciones de Pareto, con lo cual se pretende hacer frente a los diversos objetivos $\mathrm{y}$ limitaciones inherentes a la gestión de potencia en micro-redes.

\section{MODELO DE MICRORRED}

La estructura conceptual de la microrred de trabajo y sus elementos topológicos se muestran en la figura 1 . Aquí, solo se considera la generación de potencia eléctrica, y los encargados de producirla son las diferentes fuentes DER, entre las que se encuentran: los generadores diésel, biodiésel, microturbinas y celdas de combustible. Además, también se tiene en cuenta la participación de algunas fuentes de energías renovables AEDG como los generadores eólicos, fotovoltaicos y una pequeña central hidroeléctrica. Dada la condición intermitente y variable inherente a estas fuentes AEDG, es conveniente contar con un sistema de almacenamiento DS de energía. La tabla I resume los principales componentes asociados a este modelo.

\subsection{Sistema de Control Jerárquico}

De acuerdo con $[12,13]$ el EMS hace parte de una estructura de control jerárquico de tres niveles (Figura 2), en donde cada nivel sería el responsable de las siguientes tareas:

Control primario. Las cuestiones de regulación de cada elemento controlable -por ejemplo: generadoresestán integradas en este nivel. Aquí se busca regular el voltaje y la frecuencia, el intercambio de potencia activa y reactiva entre los sistemas distribuidos, a fin de garantizar que estos se puedan integrar a la microrred, logrando una estabilidad y un buen desempeño general dentro de la misma.

Control secundario. Es usado con el fin de compensar o corregir cualquier error posterior a los transitorios, producido por el control primario. Es decir, el control secundario garantiza que los niveles eléctricos se encuentren dentro de los valores requeridos. Además, es el encargado del control de sincronización para conectar o desconectar la microrred del sistema de distribución.

Control Terciario. En este nivel se encuentra el EMS, que idealmente puede ser asociado a un control de carácter "ejecutivo", embebido en el controlador central de la microrred (MGCC) [14], el cual opera y coordina una serie de DER, DS y cargas, con el fin de proporcionar energía de alta calidad, fiable y sostenible de una manera costo-efectiva. El EMS es el encargado de las diferentes estrategias y decisiones globales relacionadas con la gestión de energía, los puntos de operación de las DER, el flujo de potencia entre la microrred y la red principal, etc. Para el caso específico del presente trabajo, este sistema de gestión tiene la tarea de asignar óptimamente la potencia de salida de 


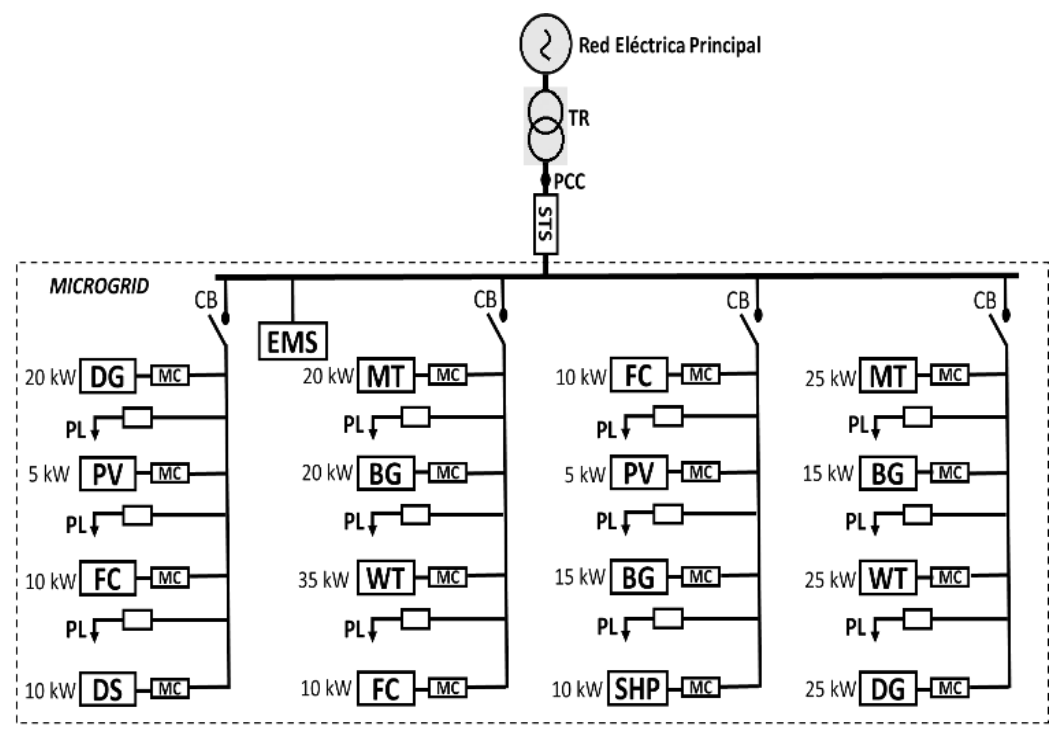

Figura 1. Estructura conceptual de la microrred de trabajo.

TABLA I.

CONVENCIONES PARA LA ESTRUCTURA CONCEPTUAL DE LA MICRORRED DE TRABAJO

\begin{tabular}{|c|c|}
\hline Elemento & Descripción \\
\hline BG & Biodiesel Generator: Generador Biodiesel \\
\hline CB & Circuit Breaker: Interruptor de Circuito \\
\hline DG & Diesel Generator: Generador Diésel \\
\hline DS & Distributed Storage: Sistema de almacenamiento distribuido \\
\hline EMS & Energy Management System: Sistema de Gestión de Energía \\
\hline FC & Fuel Cell: Celda de Combustible \\
\hline MC & Micro-source Controller: Controlador de la Microfuente \\
\hline MT & Micro-turbine: Micro Turbina \\
\hline PCC & Point of Common Coupling: Punto Común de Acoplamiento \\
\hline PL & Power Load: Carga Eléctrica \\
\hline PV & Photovoltaic Array: Arreglo Fotovoltaico \\
\hline SHP & Small Hydro-Power: Pequeña Hidroeléctrica \\
\hline STS & Static Transfer Switch: Interruptor Estático de Transferencia \\
\hline TR & Transformer: Transformador de Distribución \\
\hline WT & Wind Turbine: Generador Eólico \\
\hline
\end{tabular}


cada una de las unidades DER, en función de los costos económicos y las emisiones ambientales, esta operación se describe a partir del algoritmo mostrado en la figura 3.

\subsection{Tecnologías AEDG}

Las fuentes de energías renovables para la generación de la electricidad se visualizan como una alternativa de gran importancia en la actualidad. Son consideradas en este trabajo con un costo cero, tanto en su mantenimiento como en su operación. En las siguientes secciones se hará una breve revisión de las tecnologías utilizadas en el modelo de microrred.

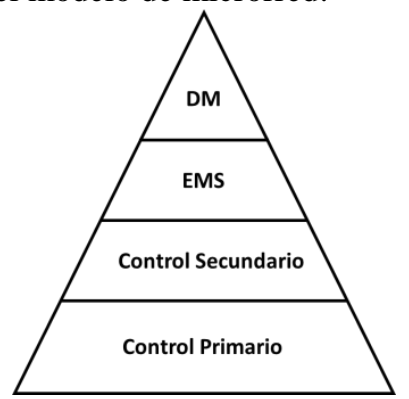

Figura 2. Estructura de control jerárquico de una microrred. Fuente: [6]

3.2.1. Generador eólico-WT. Teniendo en cuenta las características del viento, además de las características propias de una WT, la potencia de salida puede ser calculada de acuerdo a la ecuación 1, [15].

$$
\left\{\begin{array}{lr}
P_{w}=0 & V<V_{c i} \\
P_{w}=a V^{3}-b P_{r} & V_{c i}<V<V_{r} \\
P_{w}=P_{r} & V_{r}<V<V_{C O} \\
P_{w}=0 & V>V_{C O}
\end{array}\right.
$$

Donde:

$$
a=\frac{P_{r}}{V_{r}^{3}-V_{c i}^{3}} \quad \text { (2) } \quad b=\frac{V_{c i}^{3}}{V_{r}^{3}-V_{c i}^{3}}
$$

Donde $P_{r}$ es la potencia medida y $V_{c i}, V_{c o}$ y $V_{r}$ son las velocidades de corte de entrada, corte de salida y actual del viento, respectivamente. La potencia que estará disponible en WT puede obtenerse mediante la ecuación 4.

$$
P_{W T}(t)=P_{w} A_{W} \eta N_{W T}
$$

Donde $A_{W}$ es el área total de barrido de WT, $\eta$ es el coeficiente de eficiencia de WT y de sus subsistemas acoplados (conversores, acoples, etc.) y $N_{W T}$ es el número de aerogeneradores disponibles en el sitio.

3.2.2. Generador fotovoltaico-PV. La potencia de salida de un PV puede ser calculada de acuerdo con las ecuaciones 5 y 6 expuestas en [15].

$$
P_{P V}=\eta_{g} N_{P V} A_{m} G_{t}
$$

Donde $\eta_{g}$ es la eficiencia instantánea del generador $\mathrm{PV}, A_{m}$ es el área de un único módulo en $m^{2}, G_{t}$ es la radiación global incidente en el plano principal (medida en $w / m^{2}$ ) y $N_{P V}$ es el número de módulos de $\mathrm{PV}$ disponibles. La eficiencia instantánea $\eta_{g}$ puede obtenerse mediante la ecuación 6:

$$
\eta_{g}=\eta_{r} \eta_{p t}\left[1-\beta_{t}\left(T_{c}-T_{r}\right)\right]
$$

Donde $\eta_{r}$ es la eficiencia de referencia del generador $\mathrm{PV}, \eta_{p t}$ la eficiencia del sistema de seguimiento solar (igual a 1 si se garantiza un óptimo punto de radiación en todo el intervalo de generación), $T_{c}$ es la temperatura de funcionamiento de $\mathrm{PV}$ en ${ }^{\circ} C, T_{r}$ es la temperatura de referencia en la operación de PV y $\beta_{t}$ es el coeficiente de temperatura para la eficiencia.

3.2.3 Pequeña Central Hidroeléctrica-SHP. Las SHP generan electricidad mientras pase por ellas un caudal igual o superior a su mínimo técnico, de acuerdo con [16]. A diferencia de las otras tecnologías AEDG, la SHP constituye una tecnología muy bien establecida. La potencia de salida para una turbina hidráulica puede ser calculada mediante la siguiente ecuación:

$$
P=Q H \eta \rho g
$$

Donde $P$ es la potencia de salida medida en $\mathrm{W}, Q$ es el caudal del agua en $\mathrm{m}^{3} / \mathrm{s}, H$ es el desnivel en metros, $\eta$ es la eficiencia general del sistema, $\rho$ es la densidad del agua $\left(1,000 \mathrm{~kg} / \mathrm{m}^{3}\right)$ y $g$ es la aceleración gravitacional. De la anterior expresión se deduce que puede obtenerse la misma energía aprovechando grandes caudales con pequeños desniveles o pequeños caudales y grandes desniveles. 


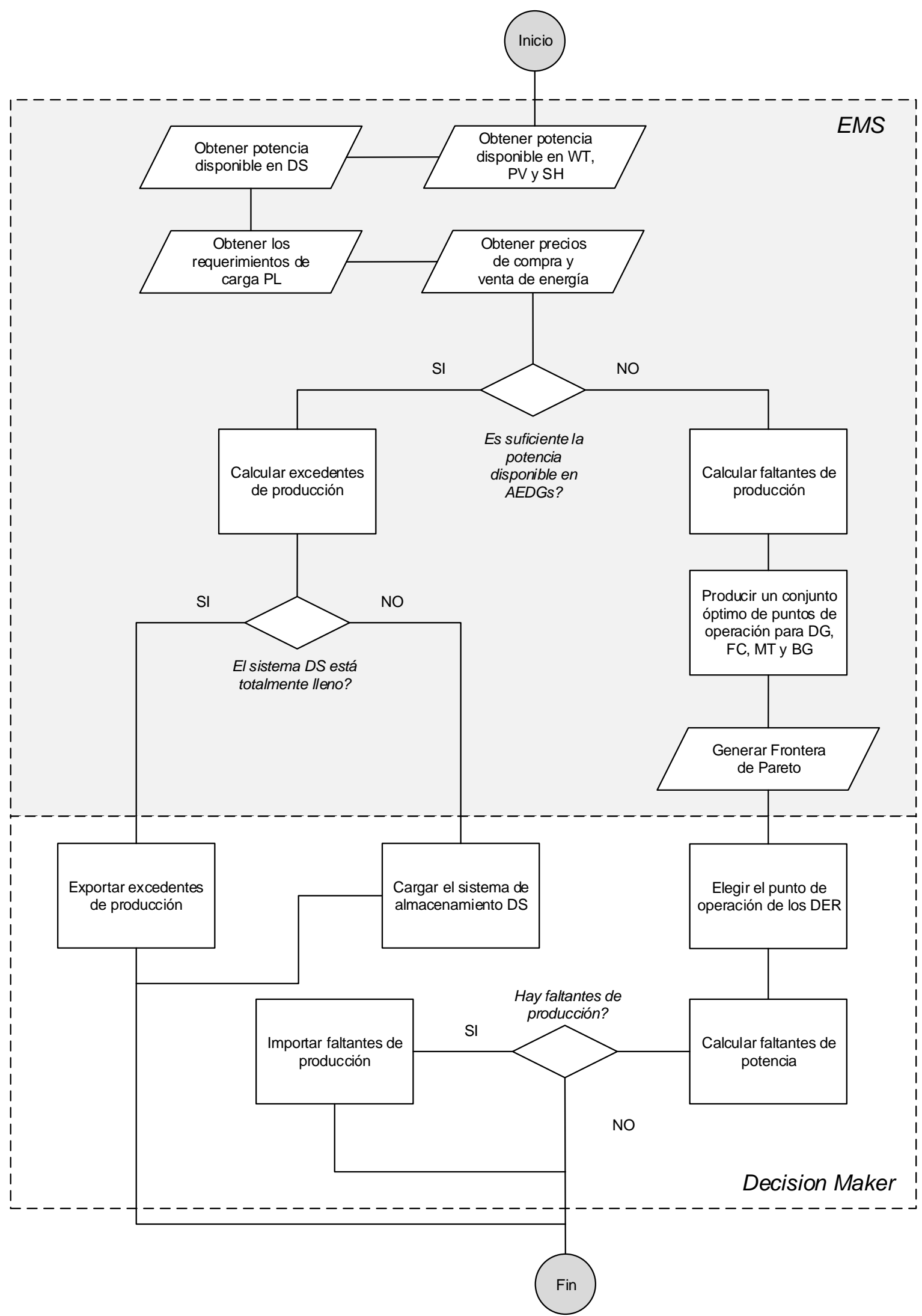

Figura 3. Algoritmo de operación del EMS+DM. Fuente: [6] 


\subsection{Sistema de Almacenamiento-DS}

Un factor importante a considerar en el diseño de una infraestructura de microrred es la condición intermitente y variable inherente a las fuentes AEDG, por lo que es conveniente contar con un sistema de almacenamiento DS de energía para suavizar la curva de disponibilidad de energía eléctrica. La ecuación 8, muestra la capacidad disponible (medida en Wh) en una hora para el banco de baterías. La ecuación 9 representa el estado de descarga cuando DS es requerida como una unidad generadora, [15].

$$
\begin{aligned}
& C_{\text {bat }}(t)=C_{\text {bat }}(t-1)(1-\sigma)- \\
& \begin{aligned}
\left(\frac{E_{\text {load }}(t)+E_{\text {grid }}(t)}{\eta_{\text {inv }}}\right. & \\
& -\left(E_{P V}(t)+E_{W T}(t)\right. \\
& \left.\left.+E_{S H P}(t)\right)\right) \eta_{\text {bat }}(8)
\end{aligned} \\
& \begin{aligned}
& C_{\text {bat }}(t)=C_{\text {bat }}(t-1)(1-\sigma)- \\
&\left(\left(E_{P V}(t)+E_{W T}(t)\right.\right.\left.+E_{S H P}(t)\right) \\
&\left.-\frac{E_{\text {load }}(t)+E_{\text {grid }}(t)}{\eta_{\text {inv }}}\right) \eta_{\text {bat }}
\end{aligned}
\end{aligned}
$$

Donde, $\quad C_{b a t}(t)$ y $\quad C_{b a t}(t-1)$ representan la capacidad disponible (medida en Wh) en el intervalo entre $t$ y $(t-1), \eta_{b a t}$ es la eficiencia del sistema DS. El parámetro $\sigma$ representa la tasa de autodescarga del banco de baterías. $E_{\text {load }}(t)$ es la demanda de carga de la microrred en una hora. $\eta_{i n v}$ es la eficiencia del sistema conversor y finalmente $E_{P V}(t), E_{W T}(t)$ y $E_{S H P}(t)$ son las energías generadas por las AEDG.

\subsection{Tecnologías DER}

Estas tecnologías presentan unas emisiones ambientales y unos costos económicos asociados a su operación, es decir, debido al costo de los combustibles como el diésel, el biodiesel, el hidrogeno y el gas natural; y a la cantidad de gases emitidos a la atmósfera como el dióxido de carbono $\mathrm{CO}_{2}$, los óxidos de nitrógeno $\mathrm{NO}_{x}$ y el óxido de sulfuro $\mathrm{SO}_{2}$.

3.4.1. Generador Diésel/Biodiesel-DG/BG. De acuerdo con [6], las tasas de consumo de combustible en DG o BG están dadas por el comportamiento natural del motor y sus cambios obedecen a variaciones de la carga. El consumo total de combustible para el i-ésimo generador diésel/biodiesel, medido en litros por hora $(1 / h)$, puede calcularse a partir de la ecuación 10 , ver [17].

$$
F_{D G, i}=\sum_{i=1}^{N} a_{i}+b_{i} P_{D G, i}+c_{i} P_{D G, i}^{2}
$$

Donde $N$ es el número de unidades DG o BG, $a_{i}, b_{i}$ y $c_{i}$ son los coeficientes que caracterizan la naturaleza del generador - usualmente entregados por el fabricante- y $P_{D G, i}(i=1,2, \ldots, N)$ es la salida de potencia del generador $i$ medida en $\mathrm{kW}$. En [18] se ve un ejemplo de los coeficientes de un generador diésel de $10 \mathrm{~kW}$, además las tasas de consumo para regímenes de $1 / 4,1 / 2,3 / 4$ y plena potencia.

3.4.2. Celda de Combustible - FC. La eficiencia práctica de una FC tipo PEMFC en estado puro de hidrógeno y oxígeno están en el rango de entre 30 y $35 \%$. La eficiencia baja cuando en lugar de hidrógeno se utilizan fuentes de hidrocarburo, metanol o gas natural. En general, la eficiencia de las FC estará dada por una relación medida en $\mathrm{kW}$ entre la potencia eléctrica de la salida y la potencia del combustible ingresado a la entrada, [19]. La ecuación 11 muestra esta relación:

$$
C_{F C}=C_{n l} \sum_{J} \frac{P_{J}}{\eta_{J}}
$$

Donde $C_{n l}$ es el precio del gas suministrado a la FC (en este caso hidrógeno), $P_{J}$ es la potencia eléctrica neta producida y $\eta_{J}$ es la eficiencia de la $\mathrm{FC}$, todo en el intervalo de tiempo $J$.

3.4.3. Micro-turbina-MT. La eficiencia total de la MT se puede obtener a partir de la ecuación 12 , como se ve en [20].

$$
\eta_{l}=\frac{P_{e l}+P_{t h, r e c}}{m_{f} * L H V_{f}}
$$

Donde $P_{e l}$ es la salida neta de potencia eléctrica, $P_{t h, r e c}$ es la potencia térmica recuperada (medida en $\mathrm{kW}), L H V_{f}$ es la tasa calórica más baja para el combustible (medida en $\mathrm{kJ} / \mathrm{kgf}$ ) y $m_{f}$ es el flujo másico del combustible $(\mathrm{kg} / \mathrm{s})$. El costo de combustible puede ser calculado de acuerdo a 13.

$$
C_{M T}=C_{n l} \sum_{J} \frac{P_{J}}{\eta_{l J}}
$$

Donde $C_{n l}$ es el precio del gas natural suministrado a la MT, $P_{J}$ es la salida neta de potencia producida en el intervalo $J$ y $\eta_{l J}$ es la eficiencia en el intervalo $J$.

\subsection{Red Pública de Energía-Main Grid}




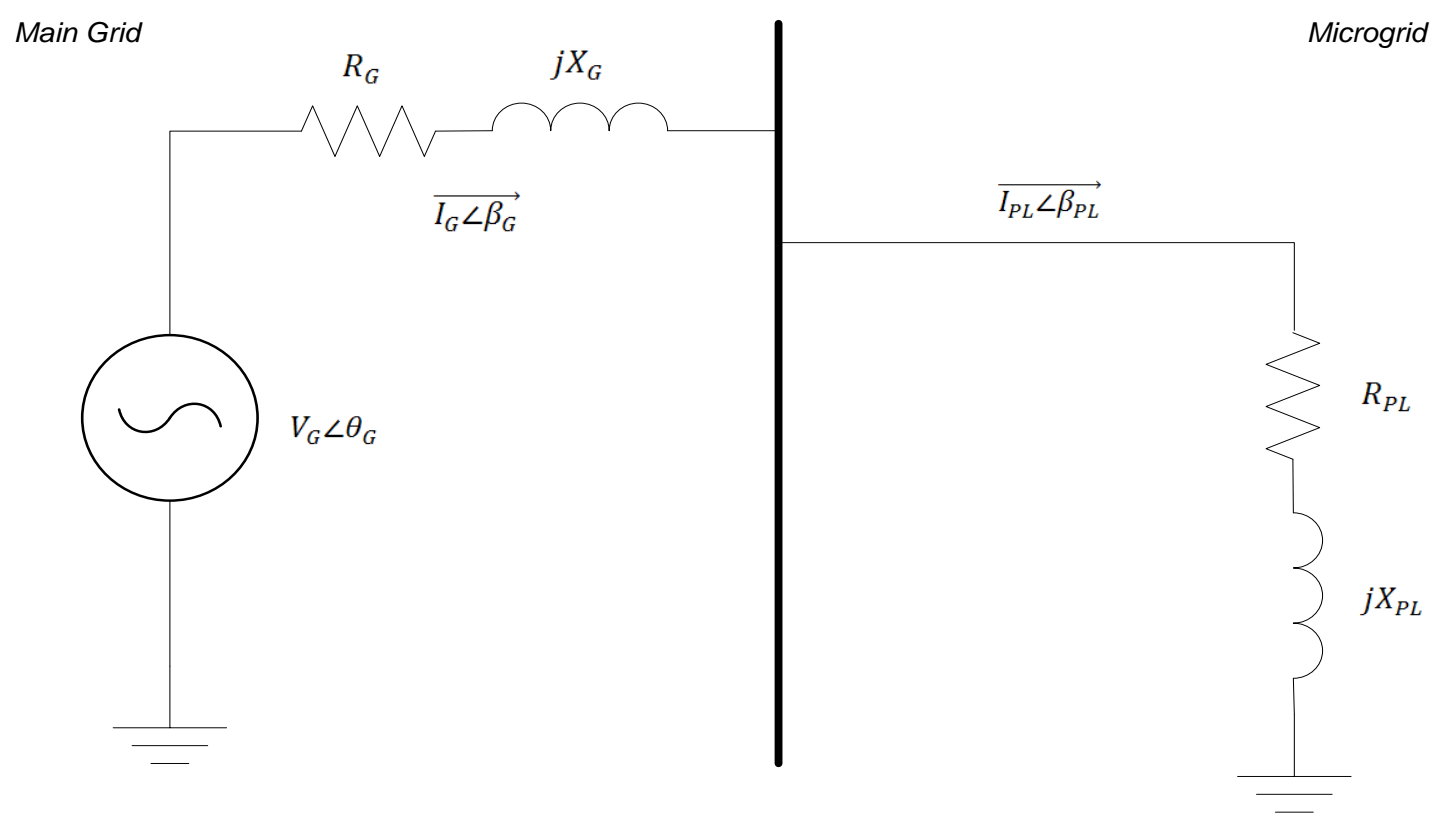

Figura 4. Esquemático general de la red eléctrica principal. Fuente: [6]

La red eléctrica principal, con generación por medio de una central térmica, [6], se modela a través del circuito de la figura 4. Las pérdidas relacionadas con la distancia pueden calcularse de acuerdo con la ecuación 14:

$$
I P_{G}=\left(\frac{E_{\text {in }}-E_{\text {out }}}{E_{\text {in }}}\right) * 100 \%
$$

Donde $I P_{G}$ representa el índice de nivel de pérdidas para la red de distribución principal, desde el punto de vista comercial, $E_{\text {in }}$ es la energía de entrada, $E_{\text {out }}$ es la energía de salida, siendo $E_{\text {out }}$ la realmente facturada, [21].

En el escenario en donde la microrred pueda interactuar con la red eléctrica principal, realizando intercambios del recurso eléctrico, el costo de comprar este recurso a la red eléctrica puede ser calculado de acuerdo a la ecuación 15 :

$$
C E I=G C_{t}\left(P D_{t}\right)
$$

Donde $C E I$ es el costo en $\$ / \mathrm{h}$ de la energía importada desde la red principal, $G C_{t}$ es la tarifa de compra de la energía en el instante $t$ y $P D_{t}$ es el déficit de potencia (medida en $\mathrm{kWh}$ ) que ha de ser importada. El valor de exportar la energía a la red principal puede ser calculado de acuerdo a la ecuación 16:

$$
C E E=G V_{t}\left(P S_{t}\right)
$$

Donde $C E E$ es el valor de venta en $\$ /$ h de la energía exportada hacia la red principal, $G V_{t}$ es la tarifa a la que la red principal está dispuesta a comprar la energía en el instante $t$ y $P S_{t}$ es la cantidad de potencia que ha de ser exportada. Estas tarifas de compra $\left(G C_{t}\right)$ y venta $\left(G C_{t}\right)$ de energía para cada hora, son mostradas en la figura 5. Los precios están dados en $\$ / \mathrm{kWh}$ y como en cualquier escenario de intercambio del recurso eléctrico, la tarifa por la energía exportada es menor que la importada.

\section{MODELO DE OPTIMIZACIÓN}

\subsection{Objetivos}

En este trabajo, la estrategia de gestión está basada en el algoritmo de optimización multiobjetivo (MOGA), que junto con unas reglas básicas de toma de decisiones (DM), tendrán la tarea de hacer una selección de los puntos de operación de las fuentes DER, con base a dos funciones objetivo. El primer objetivo es la reducción de los costos económicos asociados a la operación de la microrred $[\$ / \mathrm{h}]$. El segundo objetivo es la reducción de la cantidad de emisiones de gases de efecto invernadero y lluvia ácida, arrojados a la atmósfera por la micro-red $[\mathrm{kg} / \mathrm{h}]$. Se debe tener en cuenta que se trata de objetivos conflictivos entre sí, por cuanto maximizar los beneficios económicos puede causar significativos impactos en el medio ambiente y viceversa la reducción de emisiones contaminantes pueden incrementar los costos de operación. 


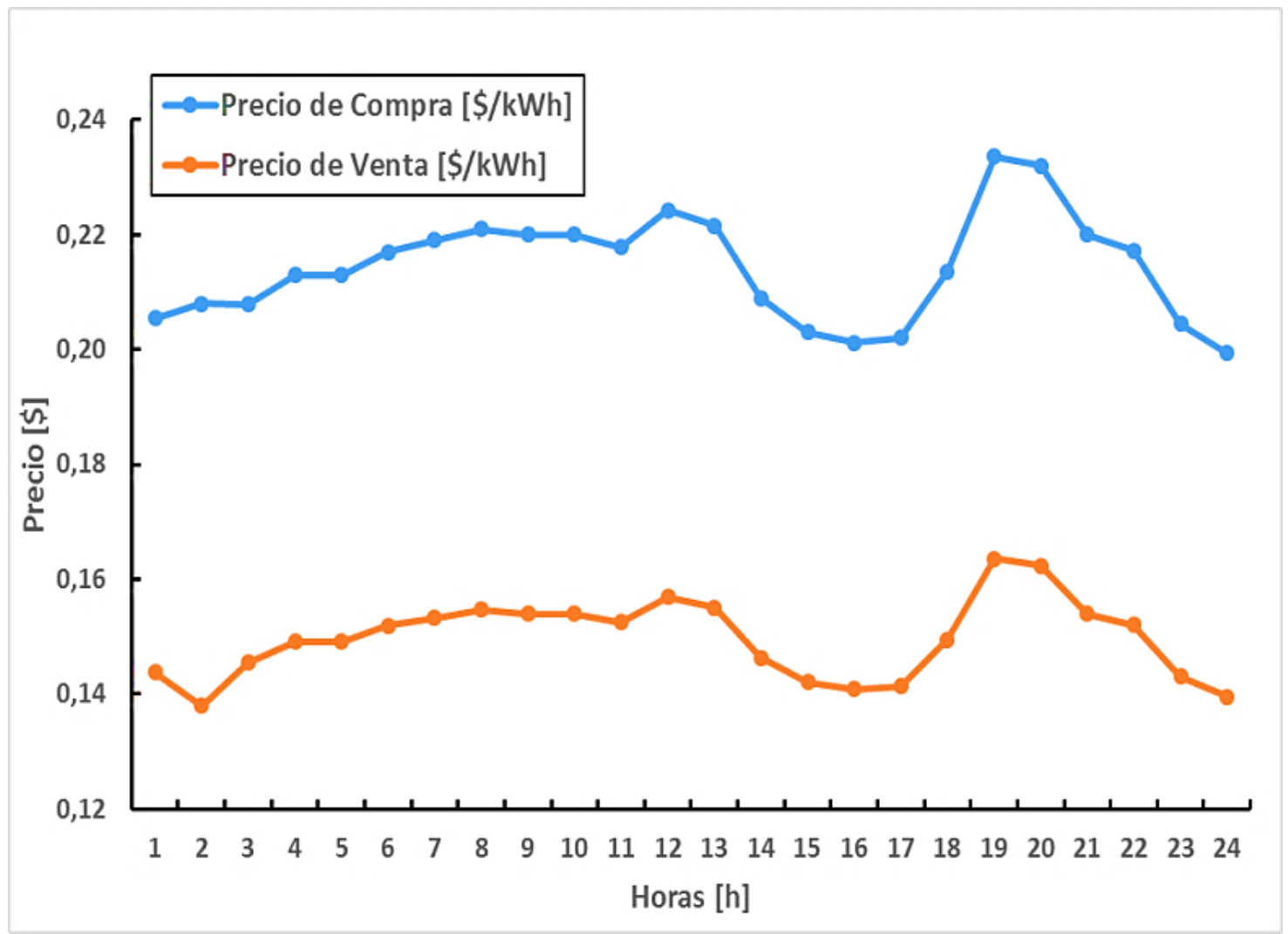

Figura 5. Fluctuación de los precios $(\$ / \mathrm{kWh})$ para la compra y la venta de energía, desde y hacia la red eléctrica principal

4.1.1 Costos económicos asociados a la operación de la microrred. Estos costos económicos son la suma de los costos de operación y mantenimiento $O M$, los costos externos, los ingresos o egresos económicos por la energía importada o exportada, el costo del combustible diésel, biodiesel, hidrogeno y gas natural, utilizados por los generadores DER.

Costos de operación y mantenimiento (OM). Están relacionados directamente con la potencia de salida generada por los DER y pueden ser calculados por medio de la ecuación 17, la cual está adaptada al contexto puramente eléctrico.

$$
O M_{i}=K_{O M} \sum_{i=1}^{N} P_{i}
$$

Donde $K_{O M}$ es la constante de O\&M medida en $\$ / \mathrm{kWh}$; su valor es diferente para cada una de las tecnologías DER. Ver Tabla II. $N$ es el número de generadores DER, $P_{i}$ es la potencia de salida del generador $i$.
TABLA II.

CONSTANTES DE COSTOS PARA LA O\&M DE DER

\begin{tabular}{|c|c|c|c|c|}
\hline & DG & BG & FC & MT \\
\hline $\boldsymbol{K}_{\text {oM }}[\mathbf{\$} / \mathbf{k W h}]$ & 0,01258 & 0,01258 & 0,00419 & 0,00587 \\
\hline
\end{tabular}

Costos externos y factores de emisión. Están asociados a las emisiones de gases de efecto invernadero y lluvia ácida $\mathrm{NOx}, \mathrm{SO} 2$ y CO2, debido a la operación de cada tecnología DER, y pueden ser calculados de acuerdo a la ecuación 18, [22]:

$$
E C=\sum_{i=1}^{N} \sum_{j=1}^{M} \alpha_{i}\left(E F_{i j} P_{i}\right)
$$

Donde $E C$ son los costos externos medidos es $\$ / \mathrm{h}$, los cuales están en función directa a la potencia de salida $P_{i}, \alpha_{i}$ es el costo externo para la emisión de tipo $j, M$ es el tipo de emisión, $i$ es el número de la unidad de generación y $N$ es el número de unidades de generación.

La tabla III muestra los coeficientes $\alpha$ para cada tipo de emisión, así como los factores de emisión para cada una de las tecnologías DER. 
TABLA III.

COEFICIENTES DE COSTOS EXTERNOS Y FACTORES DE EMISIÓN PARA LAS TECNOLOGÍAS DER

\begin{tabular}{|c|c|c|c|c|c|}
\hline $\begin{array}{c}\text { Tipo } \\
\text { de } \\
\text { emisió } \\
\text { n }\end{array}$ & $\begin{array}{c}\text { Coeficiente } \\
\text { s de costos } \\
\text { externos } \alpha \\
{[\$ / \mathbf{k g}]}\end{array}$ & $\begin{array}{c}\text { Factores } \\
\text { de } \\
\text { emisión } \\
\text { DG/GRI } \\
\text { D } \\
{[\mathbf{k g} / \mathbf{k W h}]}\end{array}$ & $\begin{array}{c}\text { Factores } \\
\text { de } \\
\text { emisión } \\
\text { BG } \\
\text { [kg/kWh } \\
\text { ] }\end{array}$ & $\begin{array}{c}\text { Factores } \\
\text { de } \\
\text { emisión } \\
\text { FC } \\
{[\mathbf{k g} / \mathbf{k W h}} \\
]\end{array}$ & $\begin{array}{c}\text { Factores } \\
\text { de } \\
\text { emisión } \\
\text { MT } \\
\text { [kg/kWh } \\
\text { ] }\end{array}$ \\
\hline$N O_{x}$ & & 0.021800 & 0.030800 & 0.000030 & 0.000440 \\
\hline $\mathrm{SO}_{2}$ & 0.990 & 0.000454 & 0.000000 & 0.000006 & 0.000008 \\
\hline $\mathrm{CO}_{2}$ & 0.014 & 0.001432 & 0.000750 & 0.001078 & 0.001596 \\
\hline
\end{tabular}

Modelo final de costos. Teniendo en cuenta los costos de los combustibles, O\&M, los ingresos o egresos, y los costos externos, la función objetivo de los costos económicos totales, queda representada por la ecuación 19:

$$
\begin{gathered}
C F(P)=\sum_{i=1}^{N}\left(C_{i} * F_{i}\left(P_{i}\right)+O M_{i}\left(P_{i}\right)+C E I-C E E\right) \\
+\sum_{j=1}^{N} \sum_{k=1}^{M} \alpha_{k}\left(E F_{j k} P_{j}\right)
\end{gathered}
$$

Donde $C F(P)$ es el costo de operación de la microrred, medido en $\$ / \mathrm{h}$. $C_{i}$ es el costo de combustible del generador $i, F_{i}\left(P_{i}\right)$ es la tasa de consumo de combustible del generador $i, O M_{i}\left(P_{i}\right)$ es el costo de O\&M del generador $i, P_{i}$ es la potencia de salida del generador $i, \vec{P}=\left[P_{1}, P_{2}, \ldots, P_{N}\right]$ es el vector de $N$ variables de decisión, $\alpha_{k}$ es el costo externo para la emisión tipo $k, E F_{j k}$ es el factor de emisión tipo $k$ en la unidad $j, M$ es el tipo de emisión y $N$ es el número de generadores DER de la microrred.

4.1.2. Emisión de gases asociados a la operación de la microrred. En las emisiones de gases contaminantes arrojados a la atmósfera por la microrred, se deben considerar aquellos gases conocidos por sus nocivos efectos sobre el calentamiento global. Entre los más conocidos se encuentra: el óxido de nitrógeno (NOx), el dióxido de carbono (CO2), y la lluvia ácida: óxido de sulfuro (SO2). En consecuencia, la función objetivo de emisión de gases queda representada por la ecuación 20 :

$$
\begin{aligned}
E\left(P_{i}\right)= & \sum_{i=1}^{N} 10^{-2}\left(\alpha_{i}+\beta_{i} P_{i}+\gamma_{i} P_{i}^{2}\right) \\
& +\zeta_{i} e^{\lambda_{i} P_{i}}
\end{aligned}
$$

Donde $E\left(P_{i}\right)$ es la cantidad de emisiones de gases NOx, SO2 y CO2 arrojados a la atmósfera, como una función de la potencia de salida de los generadores DER (medidas en $\mathrm{kg} / \mathrm{h}$ ) y $\alpha_{i}, \beta_{i}, \gamma_{i}, \zeta_{i}$ y $\lambda_{i}$ son coeficientes no negativos de las características de emisiones del generador $i$-ésimo. Ver tabla IV.
TABLA IV.

COEFICIENTES DE LAS CARACTERÍSTICAS DE EMISIÓN PARA LAS TECNOLOGÍAS DER

\begin{tabular}{|c|c|c|c|c|c|}
\hline Tecnología & $\boldsymbol{\alpha}$ & $\boldsymbol{\beta}$ & $\boldsymbol{\gamma}$ & $\boldsymbol{\zeta}$ & $\boldsymbol{\lambda}$ \\
\hline DG/BG/GRID & 0.2125 & 0.2404 & 0.2809 & $8.70^{*} 10^{-6}$ & 0.1237 \\
\hline MT & 0.0110 & 0.0120 & 0.0140 & $4.35^{*} 10^{-7}$ & 0.0062 \\
\hline FC & $0.2125^{*}$ & $0.2404^{*}$ & $0.0140^{*}$ & $4.35^{*}$ & $0.62^{*}$ \\
& $10^{-3}$ & $10^{-3}$ & $10^{-3}$ & $10^{-10}$ & $10^{-5}$ \\
\hline
\end{tabular}

\subsection{Restricciones}

Dada la naturaleza de cada uno de los componentes que conforman la microrred, las restricciones y las limitaciones de las variables de decisión tienen que ser definidas.

4.2.1. Rango de potencia de los generadores DER. El rango de potencia sobre el cual trabaja cada DER, se establece con el fin de acotar el espacio factible de búsqueda sobre el cual actúa la técnica de optimización. Además, en un modelo real de microrred, cada generador tiene un rango de trabajo establecido, que varía de acuerdo a la tecnología utilizada para su fabricación. Establecida esta condición, la restricción debida al rango de trabajo de cada generador es la que se muestra en la ecuación 21 :

$$
\operatorname{Pmin}_{i} \leq P_{i} \leq \operatorname{Pmax}_{i}, \forall i \in[1,2, \ldots, N]
$$

Donde $P \min _{i}$ es el punto mínimo de operación de la unidad $i$ y $\operatorname{Pmax}_{i}$ es el punto máximo de operación de la unidad $i$, todo medido en $\mathrm{kW}$.

4.2.2 Balance de potencia. Se considera teniendo en cuenta un escenario de operación en donde la microrred se conecta a la red principal para suplir parte de la demanda de potencia local o para venderle parte de la energía producida localmente por los DER y AEDG. Este balance está regido por la ecuación 22:

$\sum_{i=1}^{N} P_{i}=P_{L}-P_{P V}-P_{W T}-P_{S H P}-P_{D S}-P_{G}$

Donde $\sum_{i=1}^{N} P_{i}$ es la suma total de potencia generada por los DER, $P_{L}$ es el total de potencia demandada por la carga, $P_{P V}, P_{W T}$ y $P_{S H P}$ es la salida de potencia de PV, WT y SHP respectivamente, $P_{D S}$ es la potencia disponible en el sistema de almacenamiento DS, y $P_{G}$ es el déficit de potencia importada desde la red principal, todo medido en $\mathrm{kW}$. 


\section{PROPUESTA DE OPTIMIZACIÓN DE LA GESTIÓN DE POTENCIA}

En el algoritmo MOGA, [23], la población es inicializada de manera aleatoria, desde el dominio permitido a cada uno de los genes en los cromosomas, según las restricciones establecidas. El tamaño de la población se mantiene constante durante todas las iteraciones. Luego, se evalúa simultáneamente a cada individuo $x_{i}$ en las funciones objetivo, con el fin de cuantificar la calidad de una solución. Es decir, la eficiencia del cromosoma para resolver el problema, además de determinar la capacidad de un individuo para sobrevivir.

\subsection{Modelo de Datos}

En el modelo genético o cromosómico de MOGA, un cromosoma representa un vector solución, el cual está compuesto por un número de unidades discretas Gen $_{i}$ llamadas genes. Cada gen en el cromosoma representa una solución a cada variable de decisión, que en este caso son los puntos de operación $P_{i}$ de cada unidad DER. Una colección de cromosomas representa la población y cada individuo de esta población tiene una representación en el espacio de las funciones objetivo. Los datos expresados en términos del algoritmo MOGA, se muestran en la figura 6 .

\subsection{Perfil de Carga Eléctrica y Generación AEDG}

Para llevar a cabo la simulación del algoritmo, se tiene un perfil de carga eléctrica $(P L)$ como el mostrado en la figura 7, el cual está basado en un perfil presentado en [20]. El perfil utilizado muestra diferentes variaciones de la demanda con horas pico y horas valle, con lo que se pretende tener un escenario dinámico. En este escenario, la demanda de energía depende de manera directa de la carga conectada localmente a la microrred y la producción de potencia eléctrica de los generadores AEDG no es suficiente para suplirla. Por lo anterior, la estrategia de gestión basada en MOGA se emplea con el propósito de cubrir este déficit de potencia, a través de las fuentes de energía no renovable DER, siempre buscando que sus puntos de operación presenten opciones atractivas desde el punto de vista económico y ambiental.

\subsection{Estrategia para la gestión de DER}

La gestión de energía dentro del modelo de microrred depende, en gran medida, del despliegue óptimo de los DER. Los costos de operación y las emisiones ambientales dependen exclusivamente de los tipos de DER. Por lo tanto, la operación ya sea a un costo mínimo o en el nivel de emisiones mínimo, ya no puede ser una propuesta deseable. El propósito fundamental sería averiguar el compromiso simultáneo entre ambos objetivos. La estrategia de gestión aquí propuesta se generaliza más allá del proceso de optimización. Esta estrategia también comprende el tomador de decisiones DM. La decisión final sobre los puntos de operación elegidos para cada hora, será tomada por el DM, el cual deberá seleccionar una solución del conjunto de soluciones entregado por la técnica MOGA.

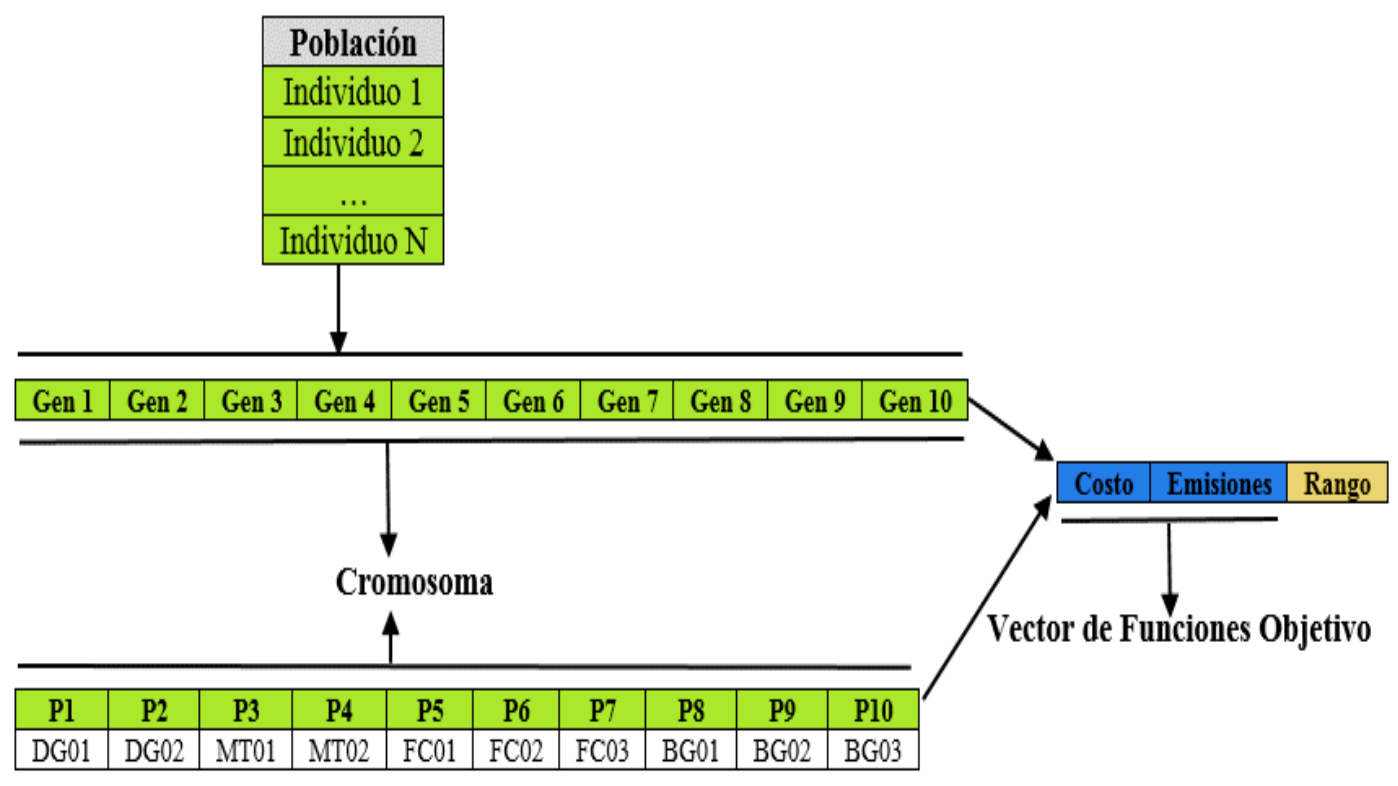

Figura 6. Modelo de datos para la optimización por medio del algoritmo MOGA 
Algoritmo 1 (MOGA Pseudocódigo)

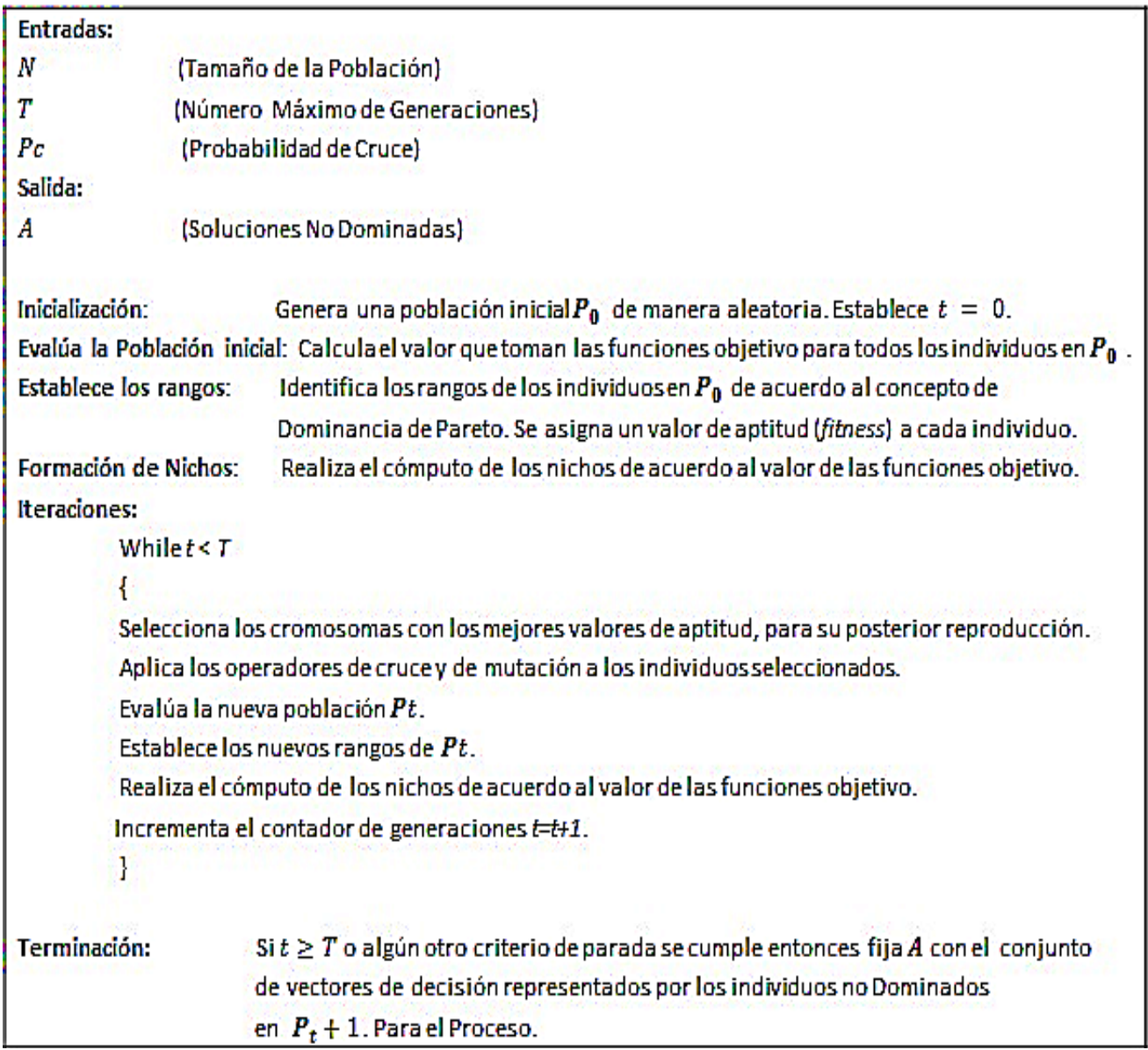


El EMS+DM tendrá la posibilidad de encontrar soluciones en las que exista un aporte de potencia de la red eléctrica principal, o incluso soluciones en las que parte de la potencia generada localmente pueda ser exportada a la red principal. Sobre la base del modo de operación y la formulación del problema de administración de energía en la microrred, la estrategia de gestión se desarrolla para lograr soluciones para cada una de las 24 horas de simulación, como se explica a continuación:

1) Para cada hora de simulación, inicialmente se debe obtener la demanda de potencia y calcular la potencia eléctrica de las fuentes AEDG, a partir de las ecuaciones 4,5 y 7 .

2) Obtener la potencia eléctrica disponible en DS, a partir de las ecuaciones 8 y 9, con el fin de decidir si este funciona como carga o como generador.

3) Obtener los precios actuales de compra y venta, para la importación o exportación de energía.

4) Calcular el déficit de potencia, como una diferencia entre la demanda y la potencia entregada por las fuentes AEDG, según lo muestra la ecuación 22.

5) Ejecutar el algoritmo MOGA, sobre los objetivos de costos económicos y emisiones ambientales, y obtener de este un conjunto de soluciones (puntos de operación de DER) y su respectiva imagen a través del vector de funciones objetivo.

6) A partir de su imagen y del conjunto de soluciones obtenidos en el paso anterior, escoger un punto de operación de los DER. Para esto, el tomador de decisiones DM utiliza el siguiente criterio de selección:

i. Calcular el costo económico de importar el déficit de potencia (obtenido en el paso 4) de la red eléctrica principal, sin tener en cuenta el aporte de los DER, llamada aquí estrategia de importación total.

ii. Seleccionar del conjunto de soluciones un punto de operación cuyo costo económico represente un valor menor (o mayor más cercano) al valor calculado en el paso i.

7) Fijar el punto de operación (seleccionado en el paso 6) a los DER que hacen parte del estudio.

8) Verificar si se puede cargar DS, con base a la producción local (DER y AEDG). En el caso de que se pueda enviar parte de la energía producida al DS, esta podría ser utilizada en la siguiente hora.

9) Verificar si se puede vender parte de la energía producida localmente a la red eléctrica principal.

10) Repetir todos los pasos anteriores para la siguiente hora de simulación.

\section{RESULTADOS}

La imagen de la población final a través del vector de funciones objetivo $F(\vec{P})$ se denomina frontera de Pareto. En la figura 8 se puede ver un ejemplo de frontera de Pareto; el eje de las abscisas corresponde a los costos económicos y el eje de las ordenadas a la cantidad de emisiones. Todos los puntos sobre la frontera representan soluciones no dominadas, por lo que no resulta trivial distinguir que solución es mejor que otra.

Por lo tanto, es necesario realizar consideraciones de más alto nivel, por medio del tomador de decisiones DM para elegir una solución. Para cada una de las 24

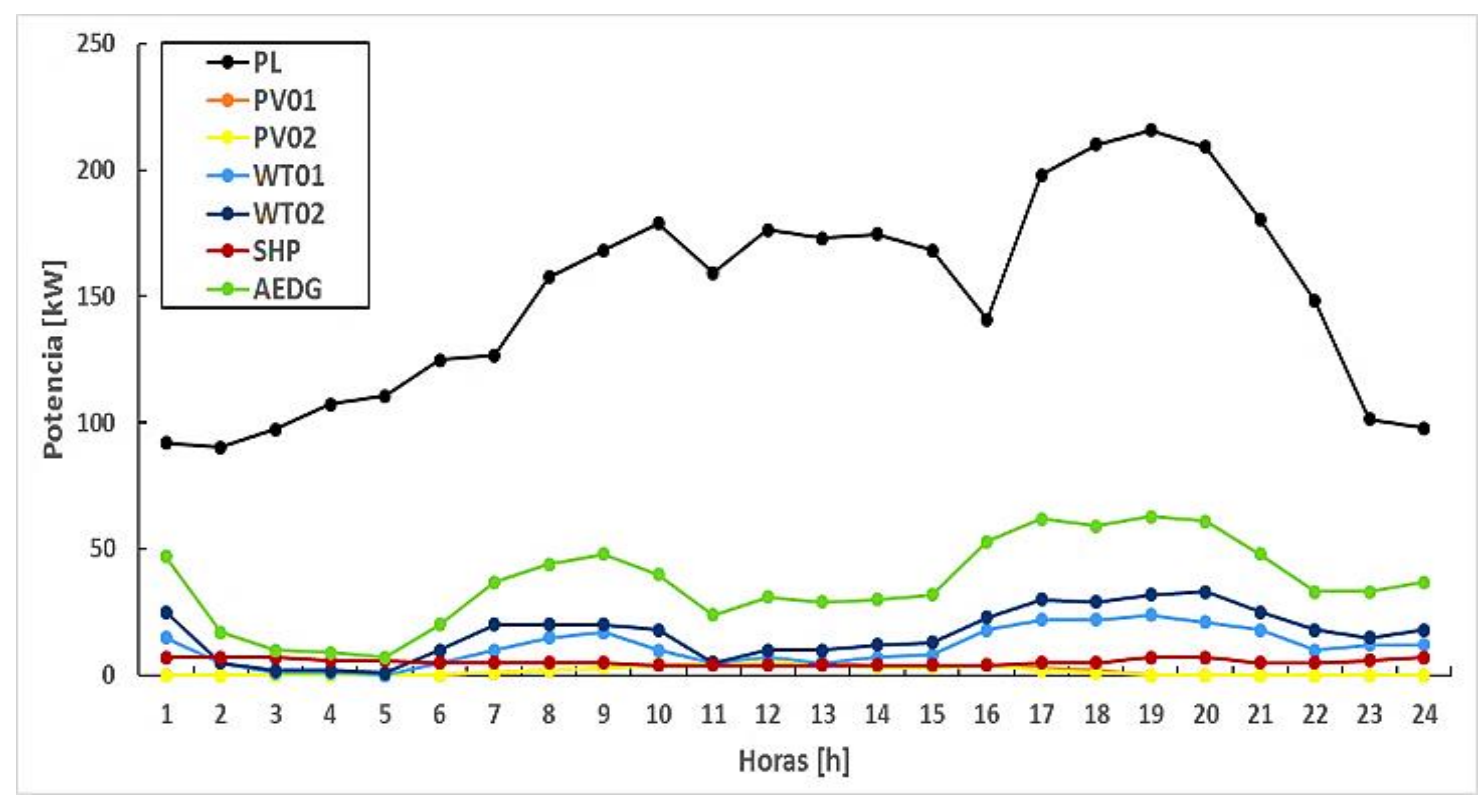

Figura 7. Perfil de carga eléctrica PL y la potencia en las tecnologías AEDG evaluadas durante 24 horas 
horas de operación de la microrred se generó un conjunto de soluciones como el de la figura 8, por

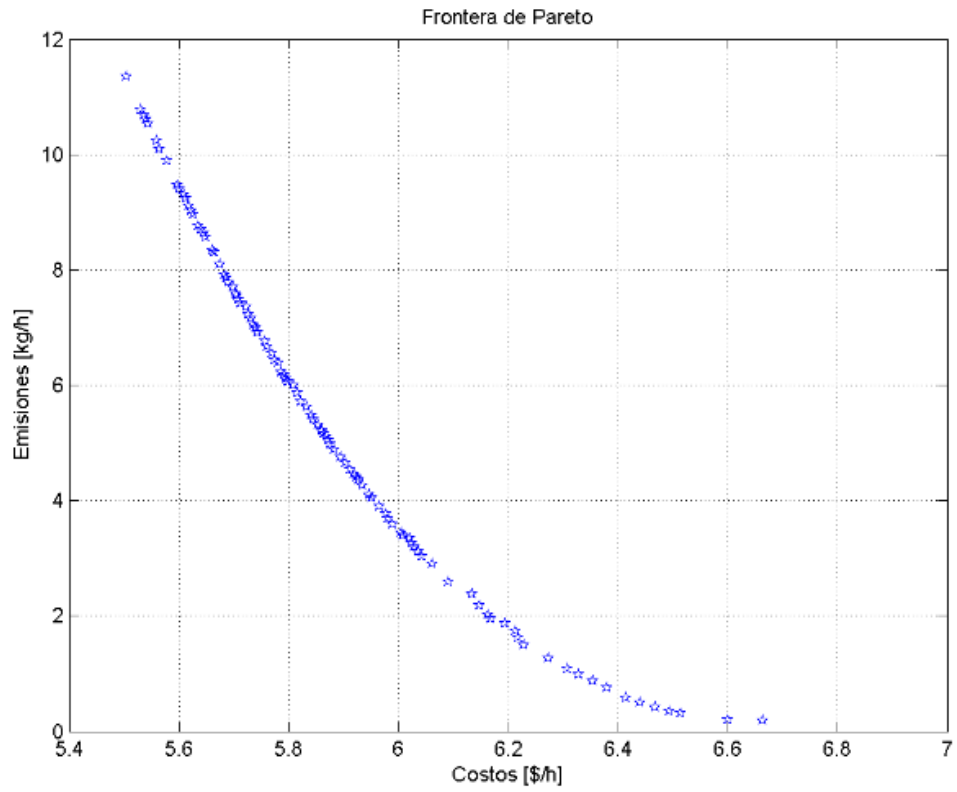

Figura 8. Ejemplo de frontera de Pareto del algoritmo de optimización MOGA obtenida en una microrred, operando con conexión a la red principal. Población $=100$, Iteraciones $=400$

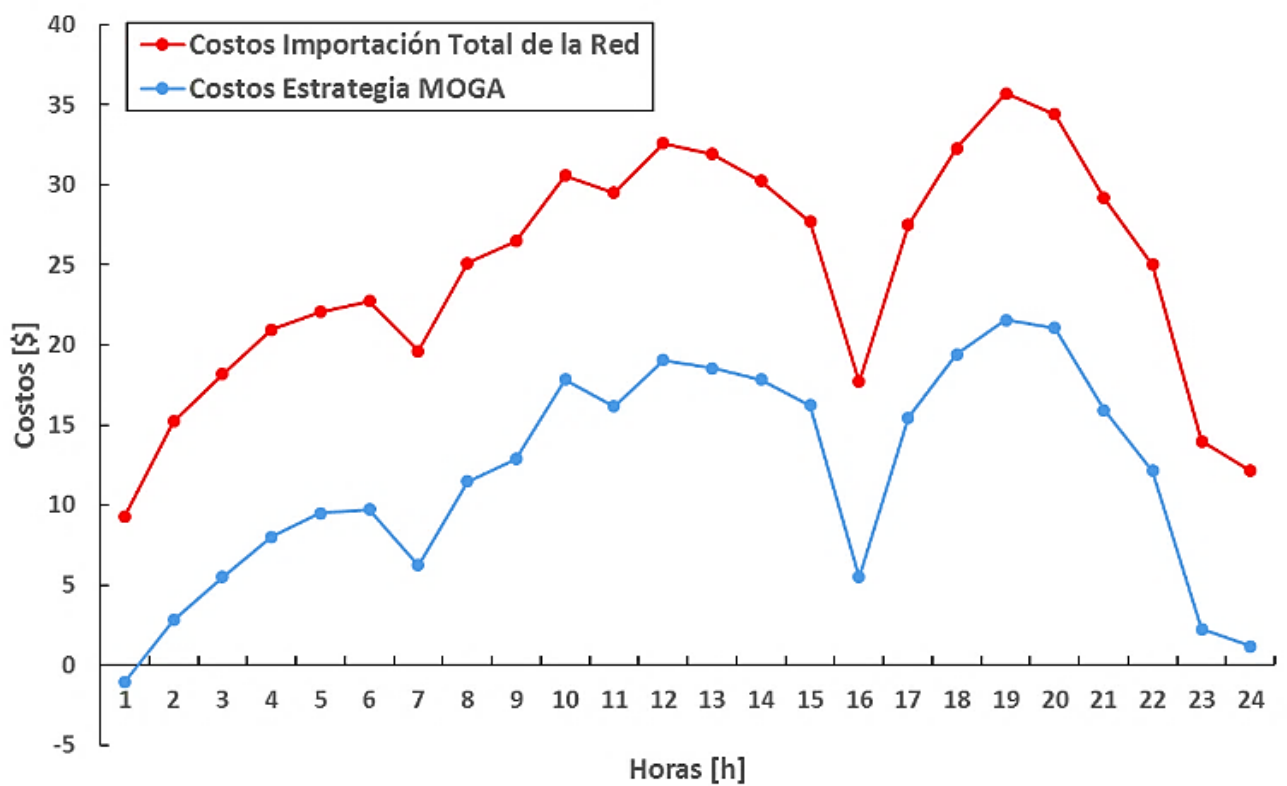

Figura 9. Comparación de los costos económicos de operación para las 24 horas entre una estrategia de importación total de energía y la estrategia MOGA 
medio de la técnica MOGA.

Dado que la microrred puede interactuar con la red eléctrica principal, realizando intercambios del recurso eléctrico, en esta sección se realiza una comparación de los costos económicos y las emisiones ambientales entre la estrategia de gestión llevada a cabo por MOGA y una estrategia de importación total de energía, en donde no existe aporte de los generadores DER.

Los resultados de costos económicos obtenidos bajo la estrategia MOGA, incluyendo el DM, son comparados con los costos generados por la estrategia de importación total; esta comparación se observa en la figura 9. Nótese como existe una reducción significativa de costos, al implementar la estrategia MOGA.

En comparación con la estrategia de importación total, esta reducción en términos porcentuales es de 51,7\%, para las 24 horas de operación (ver tabla v).

La reducción en la cantidad de emisiones de gases contaminantes entre la estrategia de importación total y la estrategia MOGA es bastante notoria, como se observa en la figura 10. Aquí, la curva de emisiones de la estrategia de importación muestra una tendencia bastante alta en las horas pico, en donde la relación entre la demanda y las emisiones presenta un comportamiento del tipo exponencial. La tabla $\mathrm{V}$ muestra la diferencia tan marcada entre las dos estrategias, con una disminución del 96,6\% por concepto de emisiones.

La tabla $\mathrm{V}$ presenta la comparación entre la estrategia de importación total y la estrategia MOGA. Aquí se observa cómo un aporte del 55,1\% de energía por parte de los DER, produce reducciones bastantes significativas en costos y emisiones.

TABLA V

COMPARACIÓN ENTRE LA ESTRATEGIA DE IMPORTACIÓN TOTAL DE LA RED Y LA ESTRATEGIA BASADA EN MOGA

\begin{tabular}{cccccc}
\hline $\begin{array}{c}\text { Estrategi } \\
\mathbf{a}\end{array}$ & $\begin{array}{c}\text { AEDG } \\
{[\%]}\end{array}$ & $\begin{array}{c}\text { DER } \\
{[\%]}\end{array}$ & $\begin{array}{c}\text { GRID } \\
{[\%]}\end{array}$ & $\begin{array}{c}\mathbf{2 4} \text { h Costos } \\
{[\$]}\end{array}$ & $\begin{array}{c}\mathbf{2 4} \mathbf{h} \\
\text { Emisiones } \\
{[\mathbf{K g}]}\end{array}$ \\
\hline $\begin{array}{c}\text { Importac } \\
\text { ión total } \\
\text { de la red }\end{array}$ & 24,2 & 0,0 & 75,8 & 589,9 & 406647,3 \\
MOGA & 24,2 & 55,1 & 21,7 & 285,1 & 13752,7 \\
\hline
\end{tabular}

La gráfica de participación de la energía mostrada en el diagrama de barras de la figura 11 da cuenta de la manera como la estrategia de optimización MOGA ofrece una tendencia a la utilización de las tecnologías DER menos contaminantes y más económicas -este es el caso de las microturbinas (MT01 y MT02) y las celdas de combustible (FC01, FC02 y FC03)-, al tiempo que reduce la participación de las tecnologías más contaminantes como las diésel (DG01 y DG02) y biodiesel (BG01, BG02 y BG03). Además, el sistema DS ha sido cargado casi cuatro veces a su máxima capacidad (como lo indica el signo negativo) durante las 24 horas de operación.

\section{CONCLUSIONES}

Se presentó una estrategia contextualizada para la gestión óptima de potencia eléctrica en una microrred, buscando la mejor solución económica y ecológica disponible, al tiempo que se cumple con los requerimientos de demanda energética. La experiencia hace énfasis en el uso de técnicas innovadoras para solucionar el problema de la gestión de potencia en microrredes. En este caso se presentó MOGA como una posible candidata para apoyar la solución. Así mismo, un modelo de microrred se presenta con todos sus parámetros de caracterización, a fin de facilitar la comparación del desempeño de técnicas similares.

La estrategia MOGA produjo una reducción significativa en costos y emisiones, en comparación con una estrategia de importación total de energía de la red principal. Esta reducción de costos en términos porcentuales fue de aproximadamente $51 \%$ para las 24 horas de operación. En cuanto a la reducción por concepto de emisiones fue bastante notoria, en un $96 \%$ aproximadamente. Con esto se destaca que la estrategia favorece el uso de tecnologías DER menos contaminantes y costosas, dentro de la mezcla disponible de GD.

Las simulaciones realizadas son una primera aproximación al funcionamiento de un sistema de gestión de energía de una microrred que contiene fuentes DER, AEDG y almacenamiento de energía eléctrica, además de una alimentación complementaria desde la red eléctrica principal. De lo anterior se puede concluir que es posible tener un gestor de energía basado en técnicas de IC, que determine un óptimo funcionamiento de una microrred. Esta metodología se podría aplicar de igual forma en otros tipos de sistemas. 


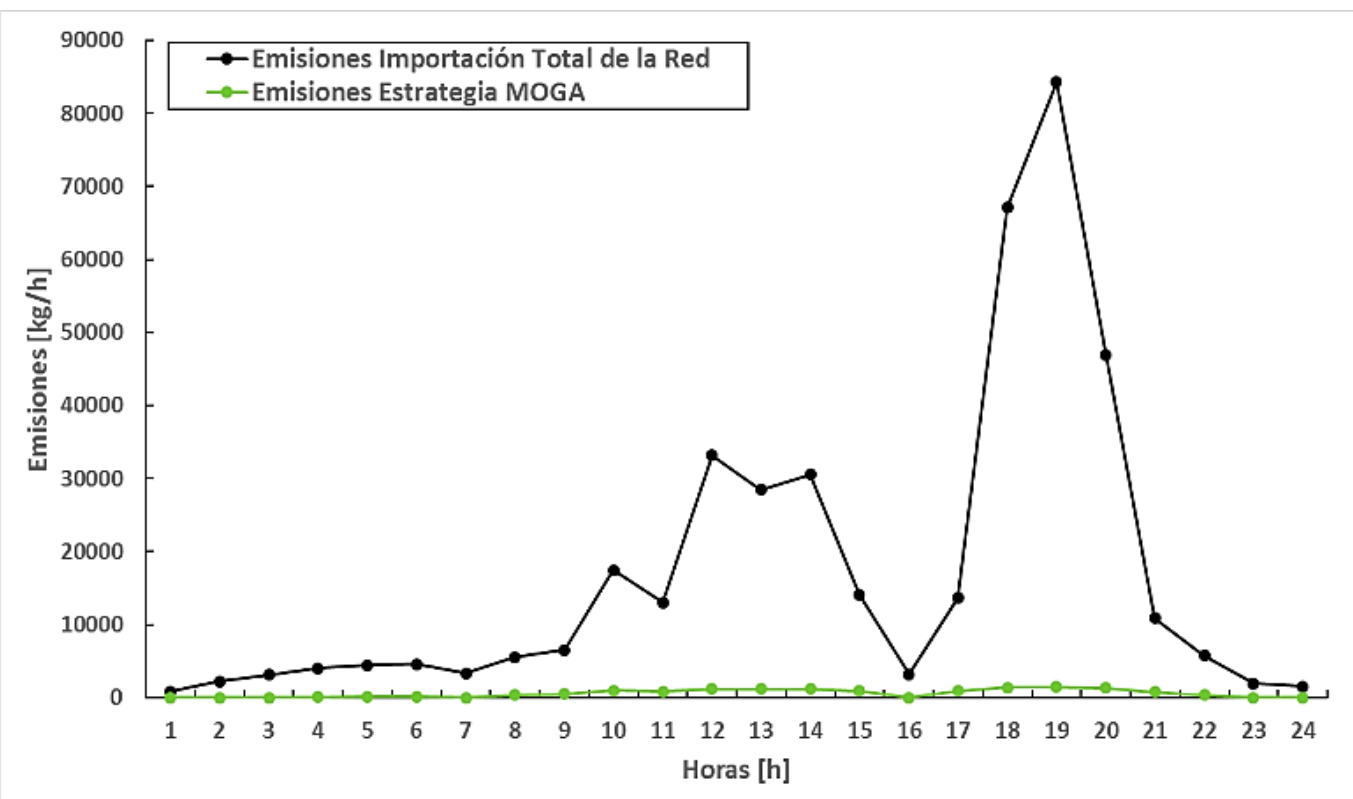

Figura 10. Comparación de las emisiones para las 24 horas entre una estrategia de importación total de energía y la estrategia MOGA.

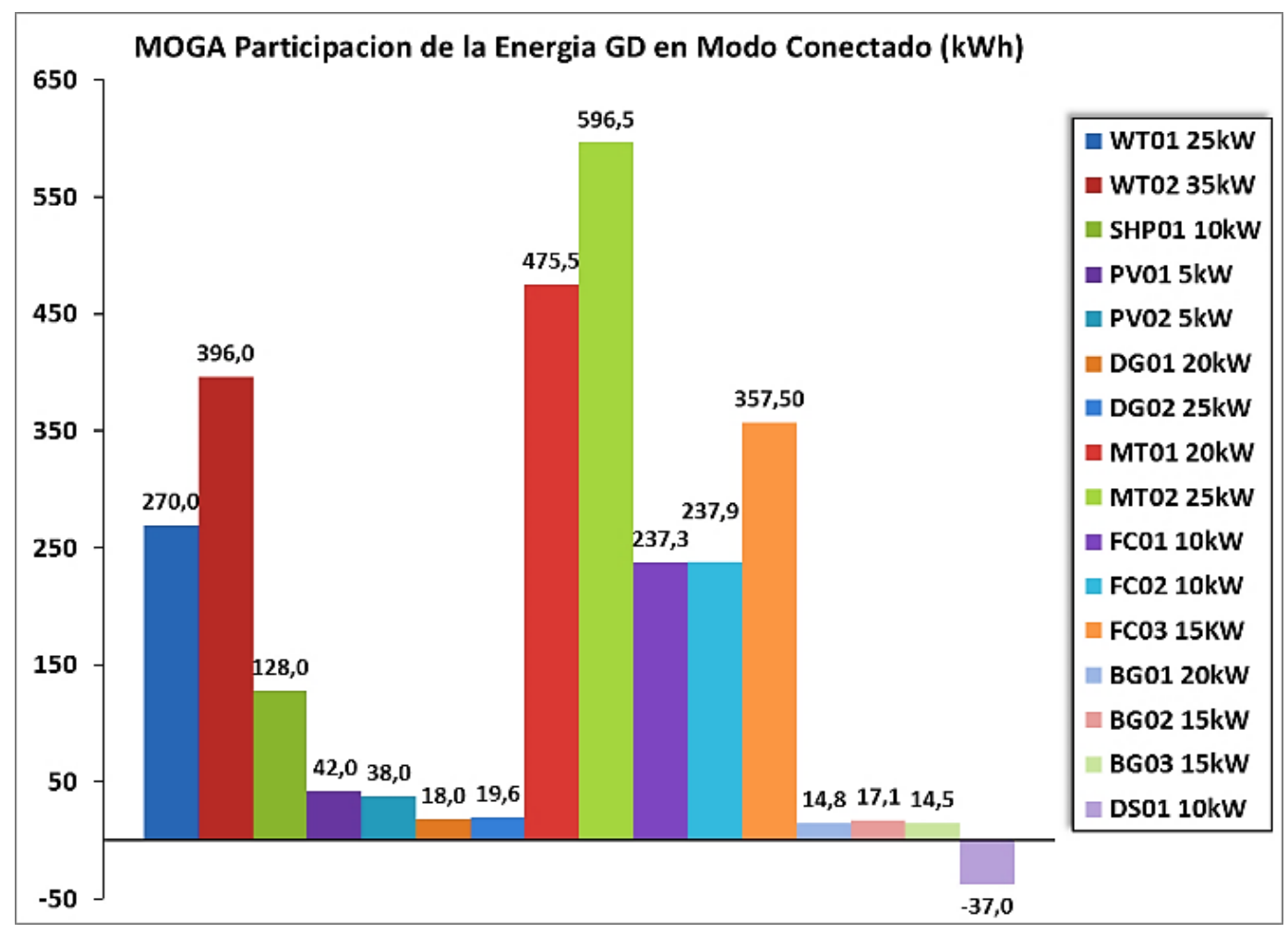

Figura 11. Participación de la energía de cada una de las tecnologías de generación bajo la estrategia MOGA 


\section{AGRADECIMIENTOS}

Los autores expresan sus agradecimientos al Departamento de Ciencias, Tecnología e Innovación Colciencias, por su apoyo financiero en el desarrollo del proyecto de investigación: "Estrategias Basadas en Inteligencia Computacional para la Gestión de la Potencia Eléctrica en Ambientes de Micro-redes". Este proyecto está enmarcado en la línea de optimización energética en la red eléctrica del futuro la Smartgrid.

\section{REFERENCIAS}

[1] R. Carballo et al., "Design and Implementation of a Three-Phase DC-AC Converter for Microgrids Based on Renewable Energy Sources," IEEE Latin America Transactions, vol. 11, núm. 1, pp. 118124, feb. 2013. DOI 10.1109/TLA.2013.6502788.

[2] Y. Muñoz y A. Ospino, "Selecting the optimal energy mix and sizing of a isolated microgrid," Revistainge@UAN, vol. 4, núm.7, pp. 59-67, juldic. 2013.

[3] G. Corso, et al. "Multi-objective long term optimal dispatch of distributed energy resources in microgrids," en UPEC, 45 th International, 2010, pp.1-5.

[4] R. Jaganmohan Reddy et al., "Distributed ANNs in a Layered Architecture for Energy Management and Maintenance Scheduling of Renewable Energy HPS Microgrids," IEEE Power and Energy Society General Meeting, 2012, pp. 1-6. DOI: 10.1109/APCET.2012.6302067

[5] P. Faria et al., "Modified Particle Swarm Optimization applied to integrated demand response and DG resources scheduling," IEEE Transactions on smart grid, vol. 4, núm. 1, pp. 606-618, mar. 2013. DOI: 10.1109/TSG. 2012.2235866.

[6] D.M. López, "Gestión óptima de la potencia eléctrica en microgrids, basada en inteligencia computacional,” tesis de maestría, Esc. Ing. Sist. Y Comp., Univalle, Santiago de Cali, 2013.

[7] M.B. Shadmand y R.S. Balog, "Multi-Objective Optimization and Design of Photovoltaic-Wind Hybrid System for Community Smart DC Microgrid," IEEE Transactions on Smart Grid, vol. 5, núm. 5, pp. 2635-2643, ago. 2014. DOI: 10.1109/TSG.2014.2315043

[8] H.A. Gabbar et al., "Performance optimization of integrated gas and power within microgrids using hybrid PSO-PS algorithm," International Journal of Energy Research, vol. 40, núm. 7, pp. 971-982, jun. 2016. DOI: 10.1002/er.3493

[9] H. Kumar Nunna y S. Doolla, "Multiagent-Based Distributed-Energy-Resource Management for Intelligent Microgrids," IEEE Transactions on Industrial Electronics, vol. 60, núm. 4, pp. 16781687, abr. 2012. DOI: 10.1109/TIE.2012.2193857
[10] M.J. Santofimia Romero, X.D.T. García y J.C. López, "Técnicas de Inteligencia Artificial aplicadas a la Red Eléctrica Inteligente (Smart Grid)," Novática, núm. 213, pp. 29-34, sep-oct 2011.

[11] European Comission, "The 2020 climate \& energy package," Disponible en: http://ec.europa.eu/clima /policies/strategies/2020_en

[12] X. Wang, J.M. Guerrero y Z.Chen, "Control of Grid Interactive AC Microgrids," IEEE International Symposium on Industrial Electronics, ISIE, jul. 2010, pp. 2211-2216. DOI: 10.1109/ISIE.2010.5637807

[13] J.M. Guerrero, J.C. Vásquez y R. Teodorescu, "Hierarchical control of droop-controlled DC and AC microgrids - a general approach towards standardization," IECON '09. 35th Annual Conference of IEEE, (Porto), 2009, pp. 4305-4310. DOI: 10.1109/IECON.2009.5414926

[14] W. Su y J. Wang, "Energy Management Systems in Microgrid Operations," The Electricity Journal, vol. 25 , núm. 8 , pp. 45-60, oct. 2012. DOI: $10.1016 /$ j.tej 2012.09.010

[15] S. Diaf et al., "A methodology for optimal sizing of autonomous hybrid PV/wind system," Energy Policy, vol. 35, núm. 11, pp. 5708-5718, nov. 2007. DOI: 10.1016/j.enpol.2007.06.020

[16] Guía para el desarrollo de una pequeña central hidroeléctrica, European Small Hydropower Association (ESHA), 2006.

[17] A.J. Wood y B.F. Wollenberg, Power Generation, Operation and Control, $2^{\mathrm{a}} \mathrm{ed}$. New York: Wiley, 1996, p. 593.

[18] Cummins Power Generation, "Generator set data sheet $10 \mathrm{~kW}$ standby DSKAA Diesel EPA".

[19] A.M. Azmy y I.Erlich, "Online optimal management of PEM fuel cells using neural networks," IEEE Trans. Power Deliv., vol. 20, núm. 2, pp. 1051-1058, abr. 2005. DOI: 10.1109/TPWRD.2004.833893

[20] R. Noroozian y H. Vahedi, "Optimal management of MicroGrid using bacterial foraging algorithm," $18^{\text {th }}$ ICEE, 2010, pp. 895-900. DOI: 10.1109/IRANIANCEE. 2010.5506951

[21] Metodología para definir el índice de perdidas reconocidas en la actividad de distribución, Comisión de Regulación de Energía y Gas (CREG), 2002.

[22] M. Pipattanasomporn, M. Willingham y S. Rahman, "Implications of on-site distributed generation for commercial/industrial facilities," IEEE Trans. On Power Systems, vol. 20, núm. 1, pp. 206-212, feb. 2005. DOI: 10.1109/TPWRS.2004.841233 
[23] C.M. Fonseca y P.J. Fleming, "Genetic Algorithms for Multi-Objective Optimization: Formulation, Discussion and Generalization 5th International Conf. on Genetic Algorithms, 1993, pp. 416-423. 